\title{
A practical approach to cystic lung disease on HRCT
}

\author{
Peter Beddy • Judith Babar • Anand Devaraj
}

Received: 29 August 2010 /Revised: 18 October 2010 / Accepted: 21 October 2010 /Published online: 12 November 2010

(C) European Society of Radiology 2010

\begin{abstract}
A lung cyst is defined as a round parenchymal lucency or area of low attenuation with a thin wall. They are not uncommon findings on high-resolution (HR) thoracic computed tomography (CT) and when identified, they require explanation. The differential diagnosis for diseases characterised by lung cysts is broad ranging from isolated chest disorders to rare multisystem diseases. This article provides a practical approach for evaluating lung cysts on HRCT, highlighting disorders in which the HRCT findings can be diagnostic as well as conditions where correlation with clinical history or biopsy is required.
\end{abstract}

Keywords Lung cyst $\cdot$ HRCT Cystic lung disease ·

Diagnostic approach

\section{Introduction}

With the exception of centrilobular emphysema, pulmonary diseases characterised by cystic air spaces are uncommon or rare conditions. However, unlike in other solid organs, the lungs do not develop so-called simple cysts. Consequently, when cysts are identified on computed tomography (CT), an underlying reason for their existence should be sought.

P. Beddy $\cdot$ J. Babar

Department of Radiology, Addenbrooke's Hospital and University of Cambridge,

Cambridge, UK

\author{
A. Devaraj $(\bowtie)$ \\ Department of Radiology, St George's Hospital, \\ Blackshaw Road, \\ London SW17 0QT, UK \\ e-mail: Anand.devaraj@stgeorges.nhs.uk
}

The differential diagnosis for lung cysts is broad and encompasses multisystem diseases in addition to cystic diseases isolated to the chest (Table 1).

The purpose of this review is to provide a practical approach to evaluating cysts when encountered on highresolution (HR) CT. The review initially deals with disorders where HRCT features may be characteristic and where the radiologist may confidently suggest a diagnosis. This is followed by a review of scenarios in which the appearances on CT are more non-specific, and where integration with clinical and/or pathological information is crucial. Disorders characterised by clustered cyst formation, such as cystic bronchiectasis or fibrotic lung disease associated with honeycombing, are unlikely to ever be in the differential diagnosis of diffuse cystic lung disease and therefore these are not discussed in this review.

The pathological basis as well as the mechanisms leading to cyst formation in the various cystic lung diseases will also be briefly discussed. At the outset it should be stressed that these mechanisms are very much speculative in nature and include a ball-valve effect causing bronchial dilatation, focal pulmonary necrosis and retractile fibrosis.

\section{Lung cysts on HRCT-how to proceed}

Pulmonary cysts should first of all be differentiated from pulmonary cavities because these two entities have very different aetiologies. The definition of a lung cyst is a round parenchymal lucency or area of low attenuation, usually thin walled (less than 2-3 mm thick) and containing air, but occasionally fluid or solid material (Fig. 1) [1]. A cavity, on the other hand, is defined as a gas-filled space which 
Table 1 Diseases that may cause pulmonary cysts

Centrilobular emphysema

Lymphangioleiomyomatosis

Langerhans cell histiocytosis

Lymphoid interstitial pneumonia

Pulmonary metastases (squamous/adenocarcinoma)

Cystic fibrohistiocytic tumour of the lung

Subacute ( \pm chronic) hypersensitivity pneumonitis

Barotrauma/ARDS

Pulmonary infection - pneumatocoeles

Desquamative interstitial pneumonia

Necrobiotic nodules (end stage)

Birt Hogg Dubé syndrome

Tracheal papillomatosis

Cystic mesenchymomas

Light-chain disease

develops in an area of pulmonary consolidation, mass or nodule (Fig. 2) [1]. Additionally, in comparison with a lung cyst, the wall of a cavity is usually relatively thick ( $>4 \mathrm{~mm}$ ). When assessing lung cysts on HRCT, the most important feature that allows differentiation between diseases is their distribution. Careful scrutiny of cyst shape and size, as well as for the presence of any ancillary findings, may help refine the differential diagnosis. Frequently, a confident diagnosis cannot be made based on CT alone and integration with clinical information is critical. Ultimately in many cases, correlation with histopathology is also required.

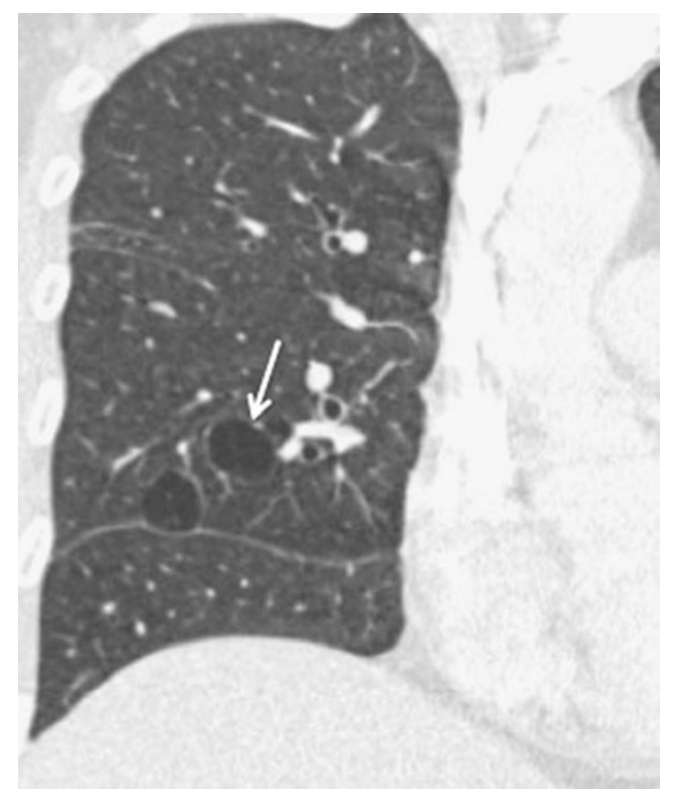

Fig. 1 HRCT image showing thin-walled cysts in the right lung (arrow) in a patient with LAM

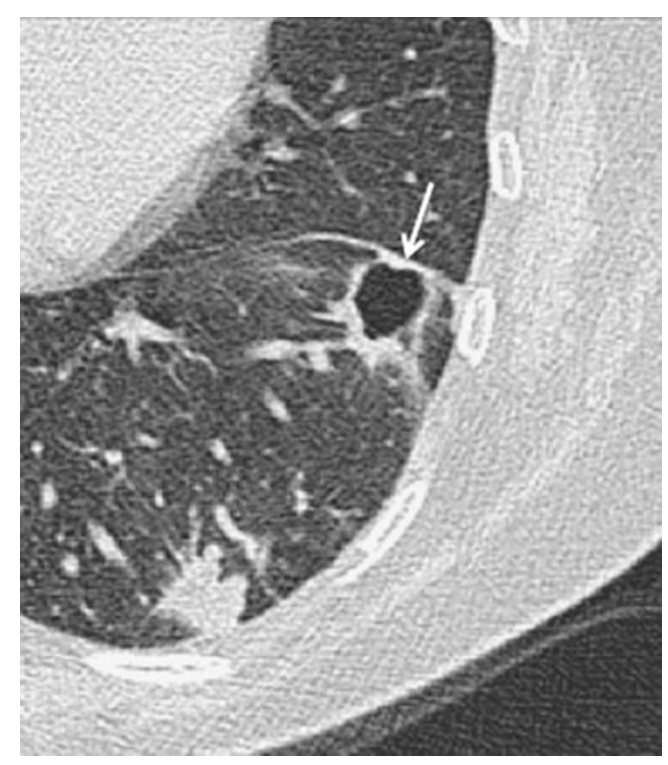

Fig. 2 HRCT shows a thick-walled cavity in the left lung adjacent to the oblique fissure (arrow) in a patient with a pneumonia. Note the thick wall and ragged edges to the cavity

\section{Scenario 1: multiple diffuse pulmonary} cysts - differentiating lymphangioleiomyomatosis (LAM), Langerhans cell histiocytosis (LCH) and centrilobular emphysema (CLE)

\section{Pulmonary LCH}

The Langerhans cell is a dendritic cell, found in many organs. Proliferation and organ infiltration of these cells results in either single or multi-organ disease [2]. Pulmonary involvement develops most commonly in isolation and occurs in young patients, between the ages of 20 and 40 years, almost all of whom are cigarette smokers. Patients may be asymptomatic or present with dyspnoea. Less frequently presenting complaints include chest pain and fever [3].

The HRCT appearances of LCH vary according to the chronicity of the disease. In the early stages, nodules (which correspond with Langerhans cell granulomas) are the predominant features, while cysts tend to develop later [4]. It has been shown that cysts in LCH probably arise because of focal dilatation of bronchi caused by destruction of small airway bronchial walls due to Langerhans cell lesions [5]. This may initially result in the nodules appearing to cavitate and a differential diagnosis for cavitating nodules may sometimes be incorrectly entertained. However, the combination of nodules, cavitating nodules and cysts in a smoker should allow a confident and accurate diagnosis to be made on CT alone [6].

The cysts in LCH also have certain relatively unique features which allow a confident distinction to be made from LAM and emphysema. The cysts are typically 
diffusely distributed, with a predominance in the lung apices and relative sparing of the lung bases [7]. Another helpful clue is observation of sparing of the medial tips of the middle lobe and lingula (Fig. 3). The cysts can have bizarre shapes and unequal sizes (Fig. 4). At this stage the cysts probably reflect LC granuloma induced fibrosis rather than bronchial dilatation.

\section{LAM}

LAM is a rare multi-organ disease characterised by infiltration of immature-appearing smooth muscle cells in the airways and along lymphatics in the chest and abdomen. It occurs either as a pure pulmonary disease or in association with tuberous sclerosis and is characterised by pulmonary cysts [8]. It is a disease that is almost exclusive to women of childbearing age and presents most commonly with dyspnoea or recurrent pneumothoraces, and occasionally haemoptysis [9].

In some patients, together with the correct clinical picture, HRCT appearances may be regarded as pathognomonic. In other instances, where clinical or radiological features are not typical, open or transbronchial lung biopsy may be required. The typical HRCT pattern is one of diffusely distributed multiple lung cysts, although the number of cysts may vary. In patients with only a few cysts, it is possible that interspaced HRCT can be 'normal' and the diagnosis made only after biopsy for the investigation of recurrent pneumothorax for example. The precise aetiology of the cysts in not clear, but it may reflect focal bronchiolar dilatation caused by a ball-valve effect due to bronchial wall smooth muscle proliferation [10].

There are some key signs that help in differentiating LAM from LCH. Perhaps the most useful differentiating

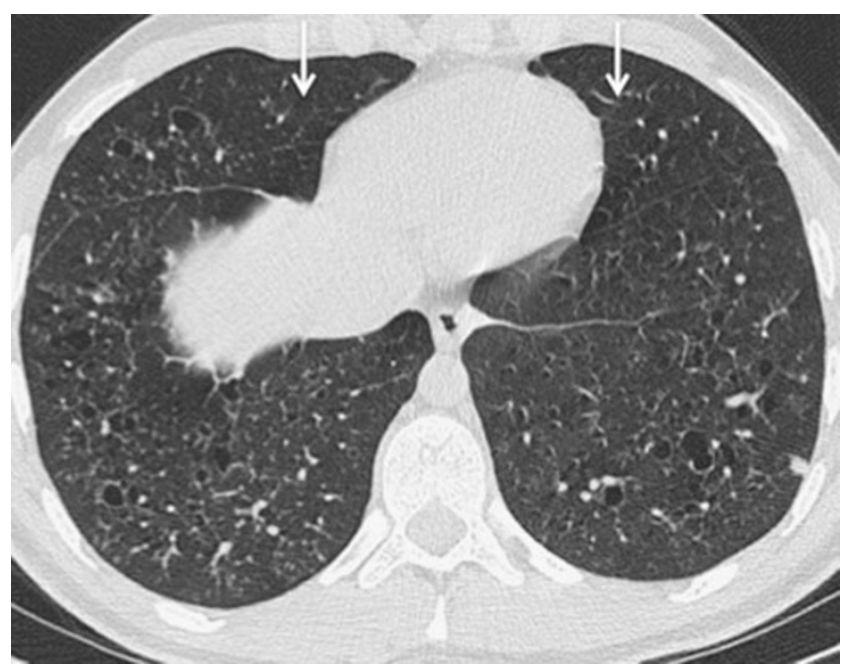

Fig. 3 HRCT demonstrates sparing of the medial segment of the middle lobe and lingula in a patient with LCH (arrows)

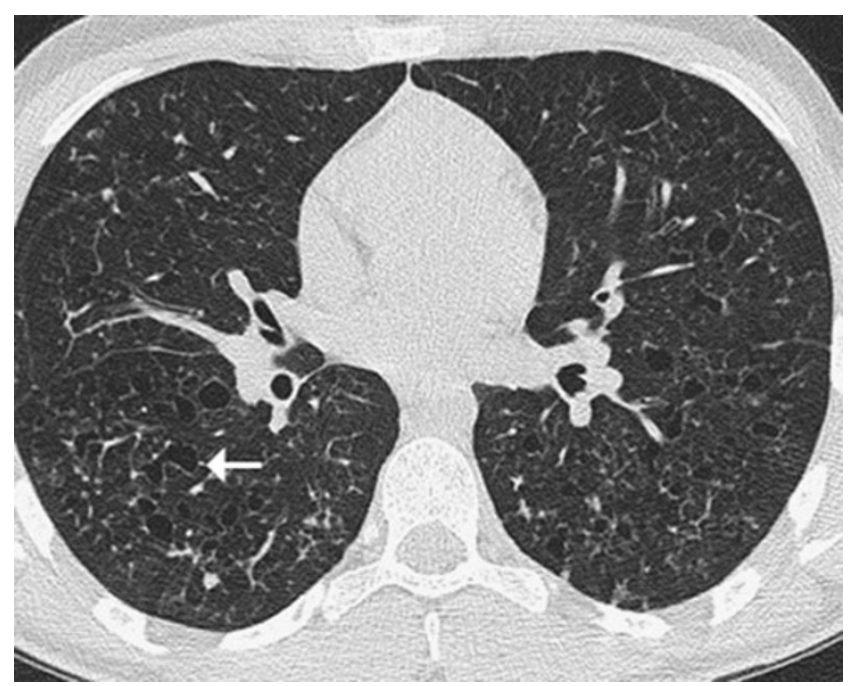

Fig. 4 HRCT of a patient with LCH. Multiple cysts are present of varying sizes and shapes (arrow)

sign is the distribution of cysts. Unlike in LCH, cysts may involve the juxtaphrenic recesses. On the other hand, there is tendency for LAM cysts to spare the extreme apices (Fig. 5) [11]. In contrast to $\mathrm{LCH}$, the intervening lung parenchyma is usually normal, although super-added ground glass opacities have been reported [7]. The cysts in LAM are typically thin walled and round in shape (Fig. 6). Bizarre shaped cysts are less common in LAM than $\mathrm{LCH}$ and tend to be seen in more end-stage disease [12].

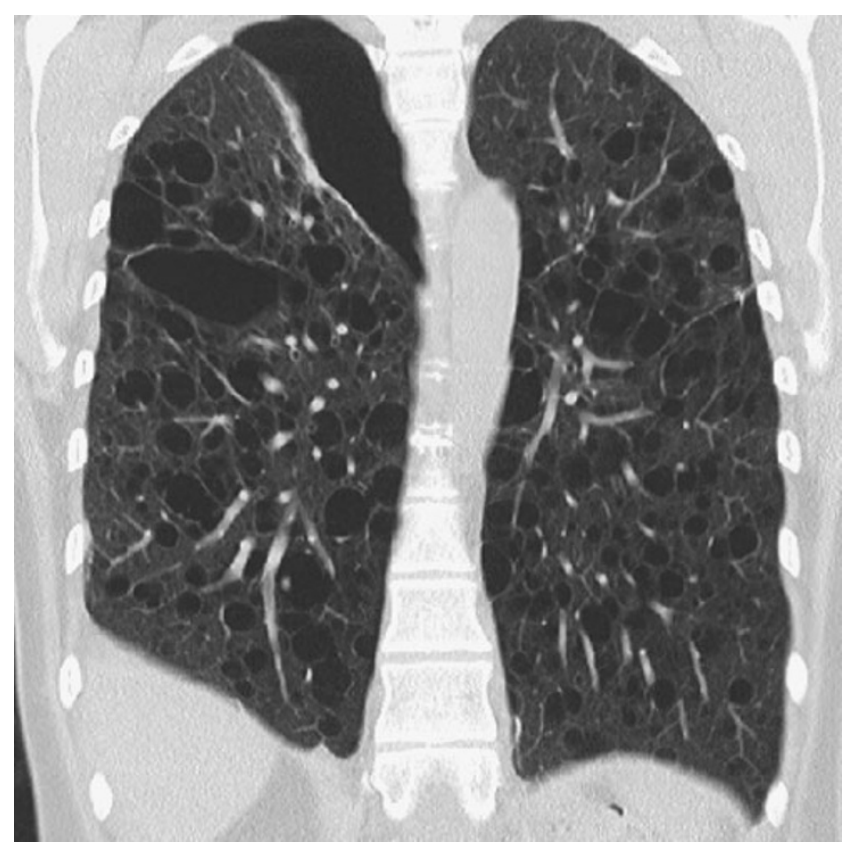

Fig. 5 HRCT of a patient with LAM and right pneumothorax. Coronal reformat demonstrates cystic involvement of the juxtraphrenic recesses, but sparing of the extreme apices 


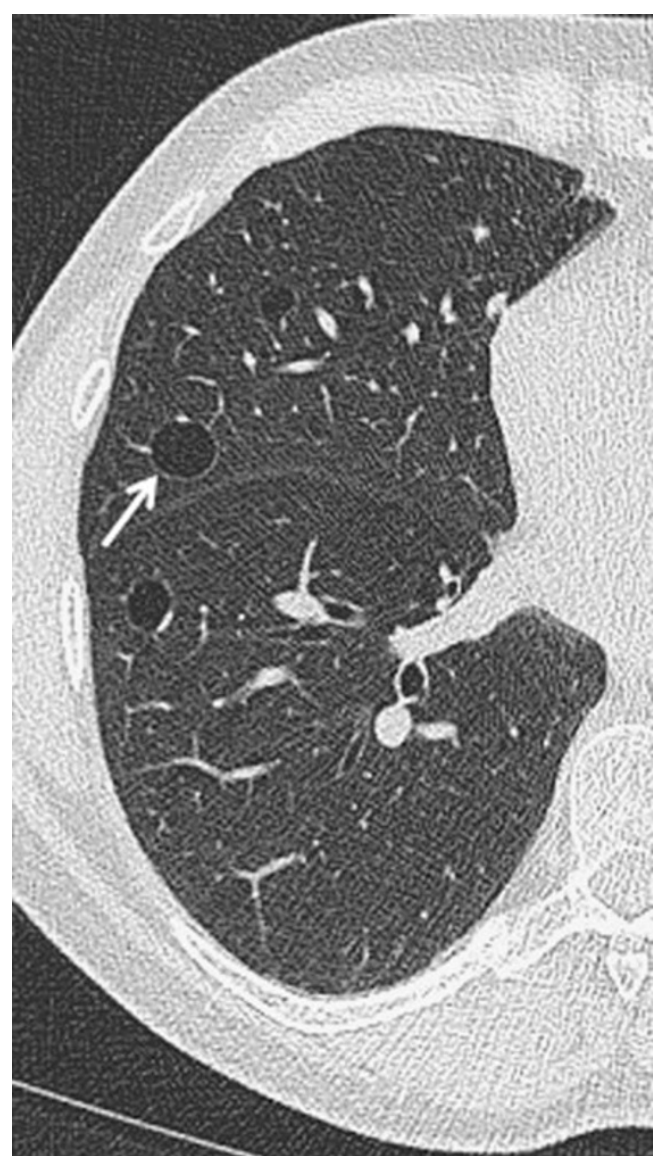

Fig. 6 HRCT shows three cysts in the right lung of a patient with LAM. Note the regular conformity of the cysts (arrow) with a thin wall

\section{CLE}

Areas of CLE are typically not surrounded by a wall and the diagnosis of CLE on CT is usually not challenging. However, CLE may appear as thin walled cysts and on occasion can be very difficult to distinguish from LAM [11]. Looking for certain CT features may help discriminate between these conditions. CLE is characterised by the presence of multiple rounded areas of low attenuation, which have diameters of several millimetres and usually have an upper lobe predominance [13]. The presence of a central core vessel (centrilobular artery) in the low attenuation cystic air space is classical of CLE (Fig. 7), whereas the cysts in LAM are devoid of intracystic structures [6]. The converse, however, does not hold true, i.e. the absence of a core structure does not exclude CLE.

Scrutiny of the cyst wall may also help. As already mentioned, CLE characteristically has no cyst wall, whereas the cyst walls in LAM are typically 1-2 mm thick [7]. However, this sign is not sufficiently robust to be relied upon in isolation. Perceptible cyst walls in CLE are well recognised (Fig. 7), while the cysts in LAM may on rare

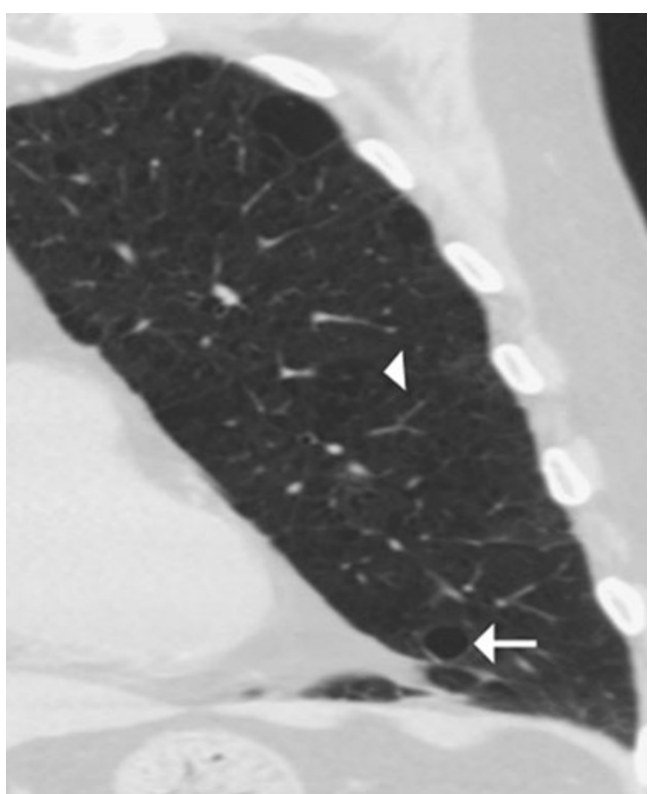

Fig. 7 Coronal reformat CT thorax in a patient with CLE demonstrates a cyst with a wall in the left lower lobe (arrow). More classical CLE with a core centrilobular artery and a much less perceptible wall is seen more superiorly (arrowhead)

occasions have no discernible wall [7]. Clearly, clinical history is of paramount importance in cases where CT is equivocal.

Scenario 2: scattered pulmonary cysts with ancillary CT signs_-differentiating lymphocytic interstitial pneumonia (LIP), hypersensitivity pneumonitis (HP) and desquamative interstitial pneumonia (DIP)

Cysts are a frequent finding in LIP and DIP and are occasionally found in HP. In comparison with LAM or $\mathrm{LCH}$, cysts in these conditions are not as profuse and so the distribution of disease does not help in refining the differential diagnosis. Instead, careful scrutiny for ancillary features, such as nodules and ground-glass opacities, is required if a diagnosis is to be suggested on radiological grounds. While nodules are a well recognised feature of $\mathrm{LCH}$, ground glass opacification is not typically associated with either LCH or LAM.

\section{LIP}

LIP is an uncommon, benign, lymphoproliferative disorder characterised pathologically by a diffuse lymphocytic infiltrate. It is often associated with collagen vascular disorders, in particular Sjogren's syndrome, but is also seen in patients with HIV infection [14]. Non-infection associated LIP typically presents in women in the 5th decade, with shortness of breath or cough [3]. The 
condition usually responds to treatment, but is known on rare occasions to be complicated by pulmonary fibrosis or to transform into lymphoma [15].

Ground-glass opacities and nodules are almost universal features in LIP, with cysts seen in about two-thirds of patients [16]. Interlobular sepal thickening and reticular opacities have also been reported $[7,16]$. The lung cysts are usually small (less than $3 \mathrm{~cm}$ ), thin walled and distributed in a scattered, random distribution (Fig. 8), though much larger cysts are also recognised [17].

Ultimately, most patients with suspected LIP on clinicoradiological grounds will require lung biopsy to confirm the diagnosis and in some cases to exclude low-grade lymphoma. At the same time, clinico-radiological integration with histological findings is essential, because it can sometimes be difficult for the pathologist to distinguish LIP from hypersensitivity pneumonitis and NSIP [15].

\section{Sub-acute HP}

HP is a diffuse granulomatous inflammatory disease of the lungs caused by exposure to a wide variety of agents ranging from organic particles to chemotherapy agents. Patients often present with gradually worsening dyspnoea, but because the presence of a precipitating agent is not always obvious from the clinical history, radiology has a role to play in suggesting the diagnosis. The key histological findings are bronchocentric lymphocytic infiltration and poorly formed granulomas, but these abnormalities are not always identified on lung or transbronchial biopsy, adding sometimes to diagnostic uncertainty [18].

Cysts on HRCT are seen in approximately $10 \%$ of patients with subacute HP and are usually few in number and random in distribution (Fig. 9) [19]. Despite their low frequency, the presence of cysts can be a helpful clue in making a radiological diagnosis of HP, when identified in

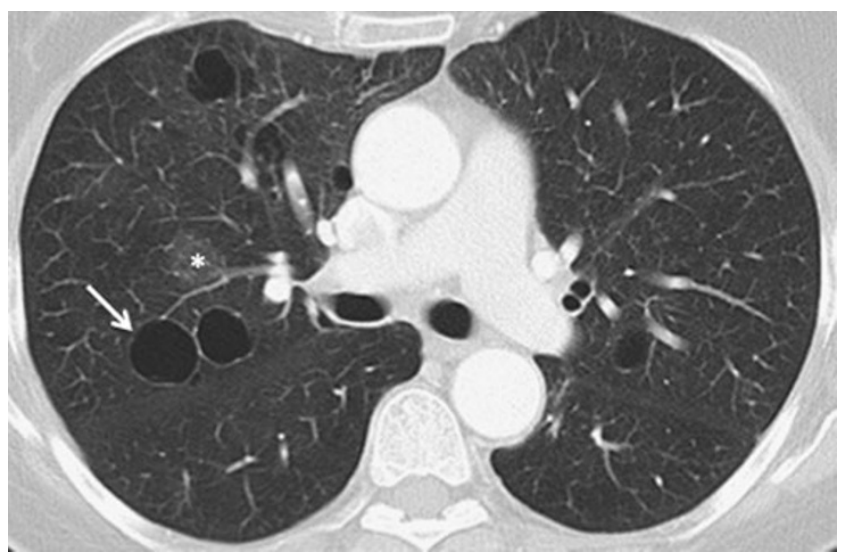

Fig. 8 HRCT shows thin walled cysts (arrow) and ground-glass opacities $\left(^{*}\right)$ in a patient with LIP. The cysts have a random distribution

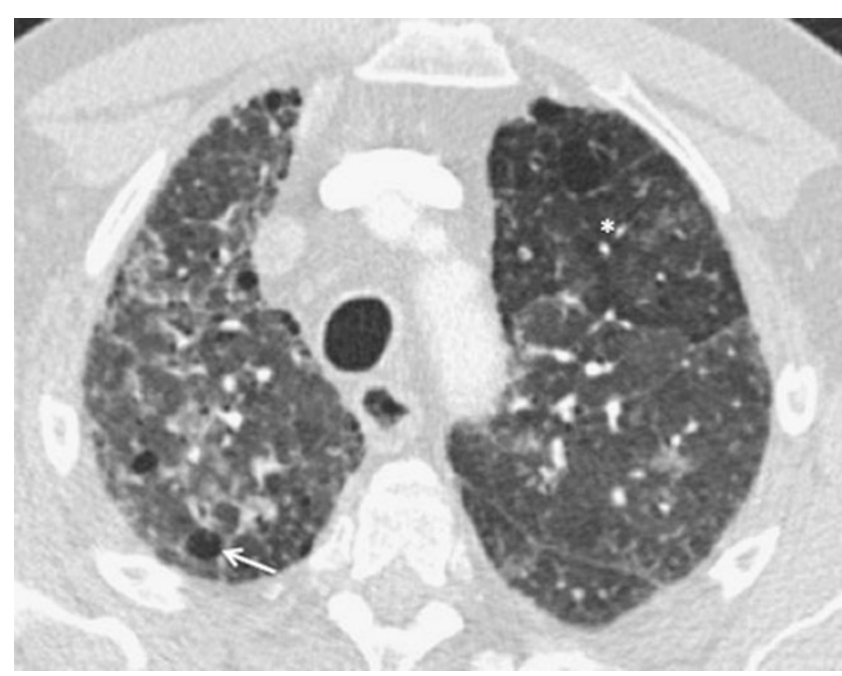

Fig. 9 HRCT from a patient with subacute HP. Small cysts are present (arrow) on a background of widespread ground glass opacity and mosaic attenuation in the anterior segment of the left upper lobe (*)

conjunction with the more classical signs of the disease: these are centrilobular ground glass nodules and a mosaic attenuation pattern. Furthermore, all of these signs of subacute HP can be seen in the more chronic fibrotic form of hypersensitivity pneumonitis [19].

Contrasting imaging features and clinical history mean that differentiating between HP and PCP (Pneumocystis jirovecii) is never usually a diagnostic dilemma. PCP in patients with AIDS commonly presents with diffuse ground glass opacification, sepal thickening and occasional cysts, but with no small airways disease [20].

\section{DIP}

DIP is an uncommon disorder characterised by macrophage accumulation within the alveoli [21]. It causes dyspnoea or cough and occurs almost exclusively in individuals exposed to cigarette smoke. Consequently, knowledge of the smoking history is mandatory before suggesting the diagnosis on radiological grounds. On HRCT, diffuse ground glass opacification is an expected finding in all patients [22]. By itself, diffuse ground-glass opacification has a wide differential diagnosis, but the identification of small cysts admixed within the ground glass opacity is a unique feature to DIP, seen in about a third of patients (Fig. 10) [23, 24].

\section{Scenario 3: isolated or scattered cysts with no ancillary CT features}

When HRCT shows just a few scattered cysts with no other abnormal features, it can be difficult to determine whether there is a true underlying cystic lung disease, or whether the 


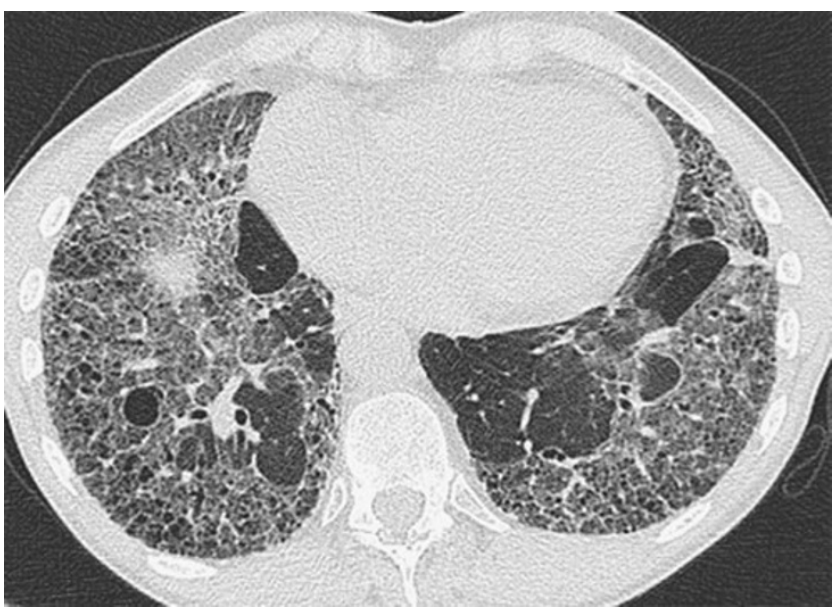

Fig. 10 HRCT showing widespread ground glass opacities and several small cysts in a patient with a strong smoking history, who was subsequently diagnosed with DIP

cysts represent an incidental and unimportant finding. Certain aspects of the clinical history may point towards a diagnosis, but ultimately lung biopsy will be required if a particular condition is suspected. For example, in a female patient who presents with a pneumothorax, a few scattered cysts may signify an early form of LAM (Fig. 11). If a family history of recurrent pneumothoraces is elicited, a diagnosis of Birt-Hogg-Dube syndrome may be suggested. This is a very rare condition that is associated with pneumothoraces, renal cell carcinomas and skin fibrofolliculomas [25]. Reports suggest that there is a lower zone preponderance for cysts in Birt-Hogg-Dube (Fig. 12) [26].

Another rare cause of lung cysts is malignancy. While cavitating malignant lung nodules are readily identified as such and do not normally pose diagnostic difficulties, very occasionally cystic metastases may present as exquisitely thin-walled cysts. Such a diagnosis should be considered if

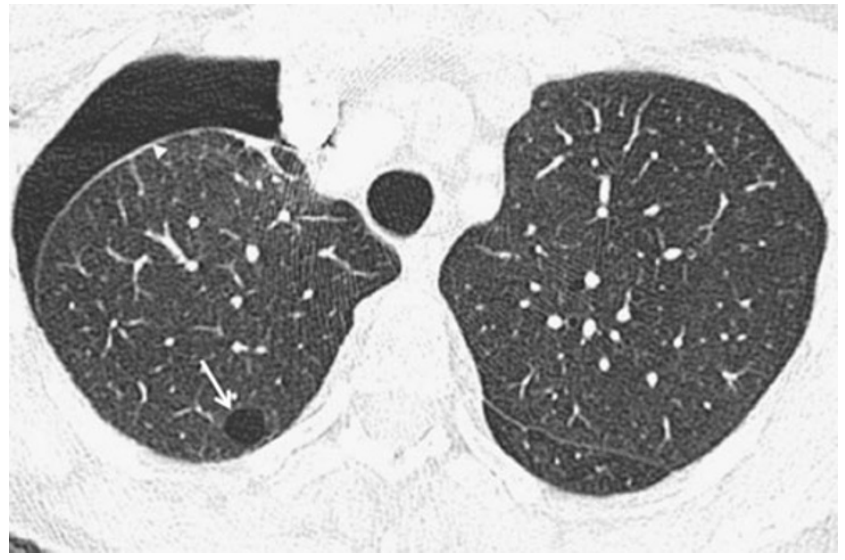

Fig. 11 HRCT of a patient presenting with a pneumothorax (arrowhead). The only parenchymal abnormality was the presence of a few small cysts, such as the one in the posterior segment of the right upper lobe (arrow). The patient was later diagnosed with LAM

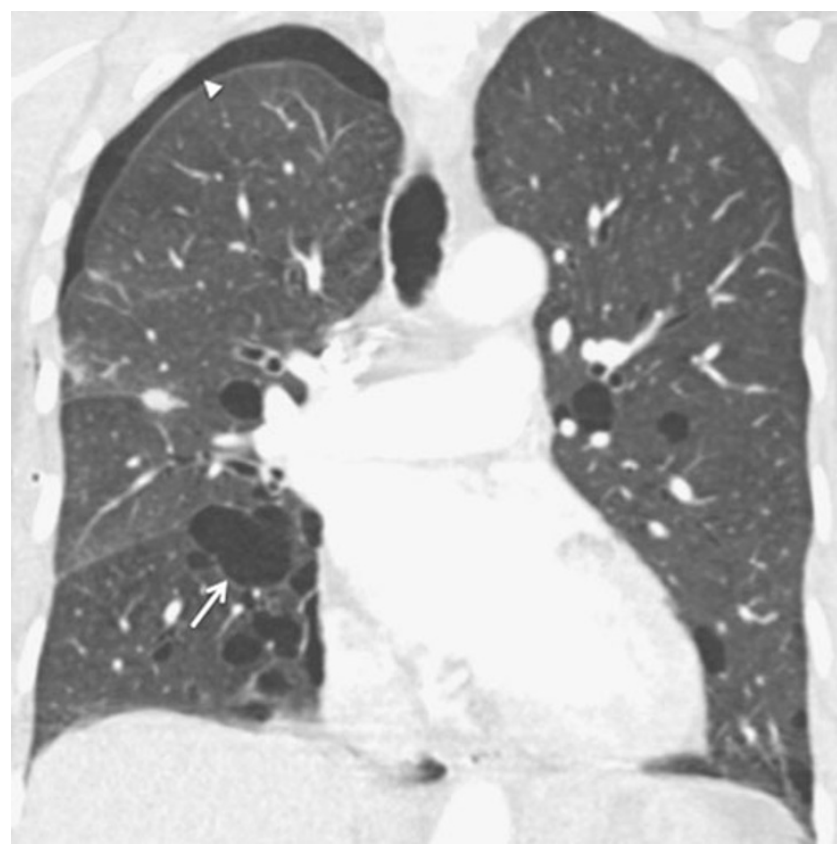

Fig. 12 Coronal reformat CT thorax shows multiple thin-walled cysts with a lower lobe predominance in a patient with Birt-Hogg-Dube. The cysts have irregular shapes (arrow) and a right apical pneumothorax is present (arrowhead). The patient had a family history of pneumothoraces

there is a history of primary squamous cell carcinoma, adenocarcinoma or sarcoma malignancies elsewhere, and in this scenario it is important not to dismiss new cystic opacities as an incidental finding (Fig. 13) [27, 28].

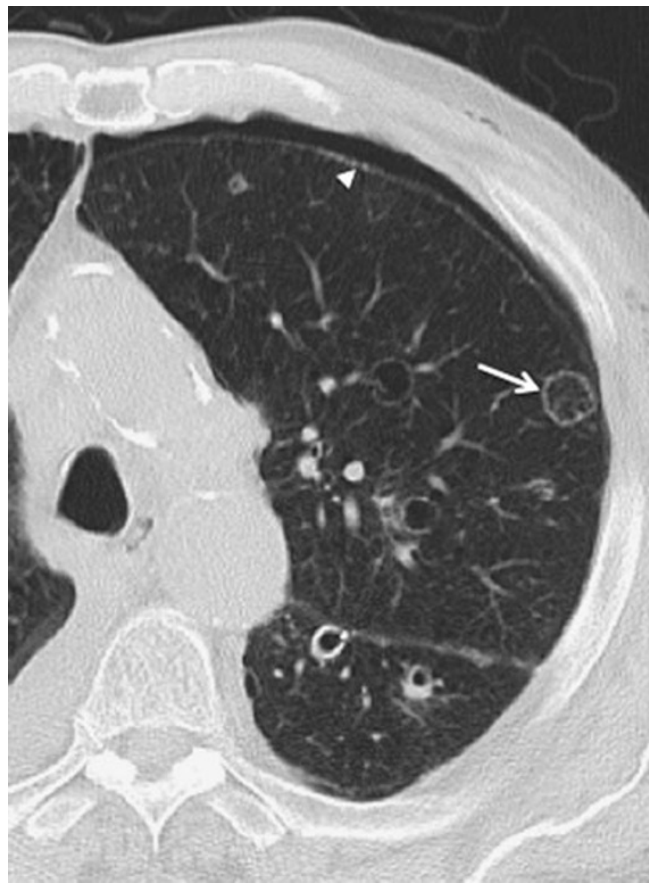

Fig. 13 Multiple cystic lesions are present in the left lung (arrow) with a small pneumothorax (arrowhead). The patient had a known history a previous oropharyngeal squamous cell carcinoma and the lesions were confirmed as cystic squamous cell carcinoma metastases 


\section{Scenario 4: the incidental lung cyst}

A solitary or small number of apparently incidental lung cysts may of course be a very early manifestation of any of the disease processes described in Table 1. However, in the absence of suspicious clinical features, further investigation is unlikely to be instigated or necessary. Nevertheless, it is important to try and explain their presence, as "simple" lung cysts are not a recognised normal phenomenon. Possible explanations include a focal area of CLE. Alternatively, a single cyst could be the residual manifestation of previous infection (i.e. a persistent pneumatocoele) [3]. In elderly patients, lung cysts have also been reported in asymptomatic non-smoking individuals, raising the possibility that they may represent part of the aging process [29].

\section{Conclusions}

Lung cysts on HRCT are not an infrequent finding and require explanation where possible. By scrutinising the distribution and shape of pulmonary cysts as well as looking for ancillary CT signs, in certain conditions HRCT is diagnostic and no further investigation is required. However, many cases will require correlation with clinical history and biopsy for a definitive diagnosis.

\section{References}

1. Hansell DM, Bankier AA, MacMahon H, McLoud TC, Muller NL, Remy J (2008) Fleischner society: glossary of terms for thoracic imaging. Radiology 246:697-722

2. Yousem SA, Colby TV, Chen YY, Chen WG, Weiss LM (2001) Pulmonary Langerhans' cell histiocytosis: molecular analysis of clonality. Am J Surg Pathol 25:630-636

3. Cosgrove GP, Frankel SK, Brown KK (2007) Challenges in pulmonary fibrosis. 3: cystic lung disease. Thorax 62:820-829

4. Soler P, Bergeron A, Kambouchner M et al (2000) Is high-resolution computed tomography a reliable tool to predict the histopathological activity of pulmonary Langerhans cell histiocytosis? Am J Respir Crit Care Med 162:264-270

5. Kambouchner M, Basset F, Marchal J, Uhl JF, Hance AJ, Soler P (2002) Three-dimensional characterization of pathologic lesions in pulmonary langerhans cell histiocytosis. Am J Respir Crit Care Med 166:1483-1490

6. Bonelli FS, Hartman TE, Swensen SJ, Sherrick A (1998) Accuracy of high-resolution CT in diagnosing lung diseases. AJR Am J Roentgenol 170:1507-1512

7. Koyama M, Johkoh T, Honda O et al (2003) Chronic cystic lung disease: diagnostic accuracy of high-resolution $\mathrm{CT}$ in 92 patients. AJR Am J Roentgenol 180:827-835

8. Raman SP, Pipavath SN, Raghu G, Schmidt RA, Godwin JD (2009) Imaging of thoracic lymphatic diseases. AJR Am J Roentgenol 193:1504-1513
9. Anton E, Casanova A, Xaubet A et al (2009) Lymphangioleiomyomatosis: a study of 72 patients from the Spanish registry. Sarcoidosis Vasc Diffuse Lung Dis 26:85-91

10. Grant LA, Babar J, Griffin N (2009) Cysts, cavities, and honeycombing in multisystem disorders: differential diagnosis and findings on thin-section CT. Clin Radiol 64:439-448

11. Rappaport DC, Weisbrod GL, Herman SJ, Chamberlain DW (1989) Pulmonary lymphangioleiomyomatosis: high-resolution CT findings in four cases. AJR Am J Roentgenol 152:961964

12. Ryu JH, Swensen SJ (2003) Cystic and cavitary lung diseases: focal and diffuse. Mayo Clin Proc 78:744-752

13. Litmanovich D, Boiselle PM, Bankier AA (2009) CT of pulmonary emphysema - current status, challenges, and future directions. Eur Radiol 19:537-551

14. Do KH, Lee JS, Seo JB et al (2005) Pulmonary parenchymal involvement of low-grade lymphoproliferative disorders. J Comput Assist Tomogr 29:825-830

15. Nicholson AG (2001) Lymphocytic interstitial pneumonia and other lymphoproliferative disorders in the lung. Semin Respir Crit Care Med 22:409-422

16. Johkoh T, Muller NL, Pickford HA et al (1999) Lymphocytic interstitial pneumonia: thin-section CT findings in 22 patients. Radiology 212:567-572

17. Silva CI, Flint JD, Levy RD, Muller NL (2006) Diffuse lung cysts in lymphoid interstitial pneumonia: high-resolution CT and pathologic findings. J Thorac Imaging 21:241-244

18. Katzenstein AL, Mukhopadhyay S, Myers JL (2008) Diagnosis of usual interstitial pneumonia and distinction from other fibrosing interstitial lung diseases. Hum Pathol 39:1275-1294

19. Franquet T, Hansell DM, Senbanjo T, Remy-Jardin M, Muller NL (2003) Lung cysts in subacute hypersensitivity pneumonitis. J Comput Assist Tomogr 27:475-478

20. Hidalgo A, Falco V, Mauleon S et al (2003) Accuracy of highresolution CT in distinguishing between Pneumocystis carinii pneumonia and non-Pneumocystis carinii pneumonia in AIDS patients. Eur Radiol 13:1179-1184

21. Attili AK, Kazerooni EA, Gross BH, Flaherty KR, Myers JL, Martinez FJ (2008) Smoking-related interstitial lung disease: radiologic-clinical-pathologic correlation. Radiographics 28:1383-1396

22. Johkoh T, Muller NL, Cartier Y et al (1999) Idiopathic interstitial pneumonias: diagnostic accuracy of thin-section CT in 129 patients. Radiology 211:555-560

23. Lee KH, Lee JS, Lynch DA, Song KS, Lim TH (2002) The radiologic differential diagnosis of diffuse lung diseases characterized by multiple cysts or cavities. J Comput Assist Tomogr 26:5-12

24. Lynch DA, Travis WD, Muller NL et al (2005) Idiopathic interstitial pneumonias: CT features. Radiology 236:10-21

25. Menko FH, van Steensel MA, Giraud S et al (2009) Birt-HoggDube syndrome: diagnosis and management. Lancet Oncol 10:1199-1206

26. Ayo DS, Aughenbaugh GL, Yi ES, Hand JL, Ryu JH (2007) Cystic lung disease in Birt-Hogg-Dube syndrome. Chest 132:679-684

27. Chaudhuri MR (1970) Cavitary pulmonary metastases. Thorax $25: 375-381$

28. Kikuchi E, Kinoshita I, Yamazaki K et al (2006) Epithelioid sarcoma presenting as pulmonary cysts with cancer antigen 125 expression. Respirology 11:826-829

29. Copley SJ, Wells AU, Hawtin KE et al (2009) Lung morphology in the elderly: comparative CT study of subjects over 75 years old versus those under 55 years old. Radiology 251:566-573 